\title{
Simple and three-valued simple minimum coloring games
}

\author{
M. Musegaas ${ }^{1}$ • P. E. M. Borm ${ }^{1}$ - M. Quant ${ }^{1}$
}

Received: 23 June 2015 / Accepted: 10 April 2016 / Published online: 27 April 2016

(C) The Author(s) 2016. This article is published with open access at Springerlink.com

\begin{abstract}
In this paper minimum coloring games are considered. We characterize the class of conflict graphs inducing simple or three-valued simple minimum coloring games. We provide an upper bound on the number of maximum cliques of conflict graphs inducing such games. Moreover, a characterization of the core is provided in terms of the underlying conflict graph. In particular, in case of a perfect conflict graph the core of an induced three-valued simple minimum coloring game equals the vital core.
\end{abstract}

Keywords Minimum coloring games · Simple games · Three-valued simple games

JEL Classification $\mathrm{C} 71 \cdot \mathrm{C} 44$

\section{Introduction}

Consider a set of agents who all need access to some type of facility, but some agents might be in conflict. All facilities are similar, but if two agents are in conflict, they cannot have access to the same facility. The total costs are linearly increasing with the number of facilities used, so the aim is to find the minimum number of facilities that can serve all agents. This problem can be modelled by an undirected graph, called the conflict graph, in which the vertices represent the agents. Two vertices are adjacent if and only if the corresponding agents are in conflict. Next, we color all vertices in such a way that any two adjacent nodes receive different colors. Finding the

\footnotetext{
$凶 \quad$ M. Musegaas

m.musegaas@tilburguniversity.edu

1 CentER and Department of Econometrics and Operations Research, Tilburg University, P.O. Box 90153, 5000 LE Tilburg, The Netherlands
} 
minimum number of facilities such that every agent has non-conflicting access to some facility, is equivalent to finding a coloring of this conflict graph that uses the smallest number of colors. This combinatorial optimization problem is known as the minimum coloring problem. A survey on minimum coloring problems can, for example, be found in Randerath and Schiermeyer (2004) and Pardalos et al. (1999). An application of the minimum coloring problem is, for example, scheduling courses at secondary schools, where some courses are compulsory and other courses are electives. Courses can be scheduled in any order, but pairs of courses are in conflict in the sense that they can not be assigned to the same time slot if there is a student who has chosen both courses.

Assuming that in the initial situation no agents share facilities, optimal cooperation in sharing facilities between non-conflicting agents will lead to minimal joint costs. To analyze how to divide the minimal joint costs among the agents, Deng et al. (1999) introduced minimum coloring games. A minimum coloring cost game can be seen as an example of a combinatorial optimization or operations research game. In a combinatorial optimization game, the value of each coalition is obtained by solving a combinatorial optimization problem on the corresponding substructure. A survey on operations research games can be found in Borm et al. (2001).

In Deng et al. (1999) the existence of core elements is investigated for the more general class of combinatorial optimization cost games where the value of a coalition is defined by an integer program. They showed that such games have a non-empty core if and only if the associated linear program has an integer optimal solution. Moreover, in case of bipartite conflict graphs, they characterized the core of the induced minimum coloring games as the convex hull of the characteristic vectors of the edges in the conflict graph. Deng et al. (2000) studied total balancedness of minimum coloring games and other combinatorial optimization games. They showed that a minimum coloring game is totally balanced if and only if the underlying conflict graph is perfect. In Okamoto (2003) concave minimum coloring games are characterized in terms of forbidden subgraphs. Moreover, for this case an explicit formula of the Shapley value is provided. In Bietenhader and Okamoto (2006) core largeness, extendability, and exactness of minimum coloring games are considered. Okamoto (2008) characterized the core of minimum coloring games on perfect conflict graphs as the convex hull of the characteristic vectors of the maximum cliques in the conflict graph, which is a generalization of the result by Deng et al. (1999). Additionally, Okamoto (2008) also investigated the nucleolus, the compromise value and the Shapley value of a minimum coloring game. The most recent work on minimum coloring games is by Hamers et al. (2014). They provided a necessary and sufficient condition for a conflict graph such that the induced minimum coloring game has a population monotonic allocation scheme.

The minimum coloring games studied in the works above are cost games. However, if we assume that in the initial situation no agents share facilities, i.e., every vertex has its own color, then cooperation in sharing facilities between non-conflicting agents will lead to cost savings. In this paper we define minimum coloring games as cost savings games instead of cost games and we focus on conflict graphs inducing coalitional cost savings in $\{0,1\}$ or $\{0,1,2\}$. Games for which the only possible values for a coalition are 0 and 1 are called simple. Simple games are introduced by von Neumann and Morgenstern (1944) and further studied by Shapley and Shubik (1954). Games for which the only possible values for a coalition are 0,1 and 2 are called three-valued 
simple. The class of three-valued simple games is a natural extension of the class of simple games. Three-valued simple games are introduced and studied by Musegaas et al. (2015). Both classes of games allow for a more explicit description of the core than general games (using the concepts of veto and vital players). Also a characterization of the Shapley value is available for these two classes of games using the transfer property. This can lead to a better understanding of how to solve, in a fair and stable way, the joint allocation problem arising from a minimum coloring problem that corresponds to a simple or three-valued simple game.

In this paper we investigate two features of simple and three-valued simple minimum coloring games. First, we characterize the class of conflict graphs inducing such games. For this characterization, a distinction is made between perfect and imperfect conflict graphs, and the concept of maximum clique is used. We show that simple minimum coloring games are always induced by perfect graphs, while three-valued simple minimum coloring games can be induced by both perfect and imperfect graphs. In particular, there is only one class of imperfect conflict graphs inducing three-valued simple minimum coloring games. We also provide an upper bound on the number of maximum cliques for conflict graphs inducing simple or three-valued simple games. Second, we characterize the core in terms of the underlying conflict graph for these games. This characterization is also based on the concept of maximum clique. Since simple minimum coloring games are always induced by perfect graphs, the characterization of the core is readily derived. We show that for three-valued simple minimum coloring games induced by imperfect conflict graphs, the core is empty. On the other hand, for three-valued simple minimum coloring games induced by perfect conflict graphs, we show that the core equals the vital core, as introduced by Musegaas et al. (2015). This strengthens the general relation between the vital core and the core for three-valued simple minimum coloring games.

The organization of this paper is as follows. Section 2 recalls basic definitions from graph theory and formally introduces minimum coloring games. In Sect. 3 simple minimum coloring games are investigated. Finally, Sect. 4 analyzes three-valued simple minimum coloring games.

\section{Minimum coloring games}

In this section we recall basic definitions from graph theory and formally define minimum coloring games. We also provide a survey of game-theoretic characteristics of minimum coloring games and in particular recall the characterization of the core of minimum coloring games associated to perfect conflict graphs.

Let $G=(N, E)$ be an undirected graph $G$, represented by a pair $(N, E)$, where $N=\{1, \ldots, n\}$ is a set of vertices and $E \subseteq\{\{i, j\} \mid i, j \in N, i \neq j\}$ is a set of edges. The graph $G$ is called complete if $E=\{\{i, j\} \mid i, j \in N, i \neq j\}$, that is every two vertices are adjacent. $K_{n}$ denotes the complete graph on $n$ vertices. For $S \subseteq N$, the subgraph of $G$ induced by $S$ is the graph $G[S]=\left(S, E_{S}\right)$ where $E_{S}=\{\{i, j\} \in E \mid i, j \in S\}$. The complement of $G$ is the graph $\bar{G}=(N, \bar{E})$ where $\bar{E}=\{\{i, j\} \mid i, j \in N, i \neq j,\{i, j\} \notin E\}$. In this paper we only consider graphs that are connected on $N$, i.e., every pair of vertices is linked via a sequence of consecutive 
edges in $E$. However, note that it still might happen that some induced subgraph $G[S]$ is not connected on $S$ via $E_{S}$. For conflict graphs, $\{i, j\} \in E$ is interpreted as $i$ and $j$ being in conflict.

A coloring of $G$ is a mapping $\gamma: N \rightarrow \mathbb{N}$ such that $\gamma(i) \neq \gamma(j)$ for every $\{i, j\} \in E$. The natural numbers assigned to the vertices correspond to the colors assigned to the vertices. A minimum coloring of $G$ is a coloring $\gamma$ that uses the smallest number of colors, i.e., a coloring for which $|\{\gamma(i) \mid i \in N\}|$ is minimal. The number of colors in a minimum coloring is called the chromatic number of $G$ and is denoted by $\chi(G)$. The problem of finding a minimum coloring of a conflict graph $(N, E)$ is called a minimum coloring problem.

The chromatic number of a conflict graph is strongly related to the concept of a clique, where a clique in $G$ is a set $S \subseteq N$ such that $G[S]=K_{|S|}$. A maximum clique of $G$ is a clique $S$ of the largest possible size, i.e., a clique for which $|S|$ is maximal. The number of vertices in a maximum clique is called the clique number of $G$ and is denoted by $\omega(G)$. We denote the set of all maximum cliques in $G$ by $\Omega(G)$, i.e.,

$$
\Omega(G)=\left\{S \subseteq N \mid G[S]=K_{\omega(G)}\right\}
$$

Note that all vertices in a maximum clique are mutually adjacent and therefore each of them has to receive a different color in a minimum coloring, so

$$
\chi(G) \geq \omega(G)
$$

for any conflict graph $G$.

With $N$ a non-empty finite set of players, a transferable utility (TU) game is a function $v: 2^{N} \rightarrow \mathbb{R}$ which assigns a number to each coalition $S \in 2^{N}$, where $2^{N}$ denotes the collection of all subsets of $N$. The value $v(S)$ denotes the highest joint monetary payoff or cost savings the coalition $S$ can jointly generate by means of optimal cooperation without any help of the players in $N \backslash S$. By convention, $v(\emptyset)=0$. Let $\mathrm{TU}^{N}$ denote the class of all TU-games with player set $N$.

If we assume that initially every vertex has its own color, then the minimum coloring of $G$ results in optimal cost savings for $N$ as a whole. To tackle the allocation problem of these cost savings one can analyze an associated TU-game $v^{G}$ to a minimum coloring problem with conflict graph $G=(N, E)$, where the set of players is the set of vertices. For a coalition $S \subseteq N, v(S)$ reflects the maximal cost savings this coalition can generate, i.e., the number of colors that are saved with respect to the initial situation where $|S|$ colors were used. Hence, the value of coalition $S$ is obtained by solving the minimum coloring problem with conflict graph $G[S]$. Correspondingly, the minimum coloring game $v^{G} \in \mathrm{TU}^{N}$ induced by the conflict graph $G=(N, E)$ is defined by

$$
v^{G}(S)=|S|-\chi(G[S])
$$

for all $S \subseteq N$.

In the following, we give an example of a minimum coloring game and discuss the concepts of clique number and chromatic number. 


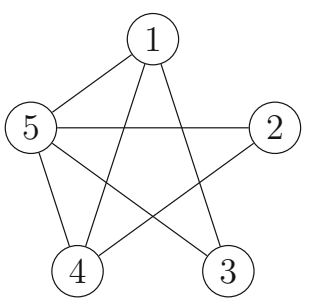

Fig. 1 The conflict graph of example 2.1

Example 2.1 Consider the conflict graph $G=(N, E)$ with $N=\{1, \ldots, 5\}$ as depicted in Fig. 1 . Note that $\Omega(G)=\{\{1,3,5\},\{1,4,5\},\{2,4,5\}\}$, so using (1) we have $\chi(G) \geq \omega(G)=3$. Consider the following coloring of $G$ with three colors given by the function $\gamma: N \rightarrow\{1,2,3\}$ with

$$
\begin{aligned}
& \gamma(1)=\gamma(2)=1, \\
& \gamma(3)=\gamma(4)=2, \\
& \gamma(5)=3 .
\end{aligned}
$$

We may conclude that this coloring $\gamma$ is a minimum coloring of $G$ and $\chi(G)=3$. As a consequence, the value of the grand coalition $N$ is given by

$$
v^{G}(N)=5-\chi(G)=2 .
$$

For coalition $\{1,2\}$, the induced subgraph $G[\{1,2\}]$ contains no edges and thus only one color is needed to color the vertices, i.e., $\chi(G[\{1,2\}])=1$. Hence, the value of coalition $\{1,2\}$ is given by

$$
v^{G}(\{1,2\})=2-\chi(G[\{1,2\}])=1 .
$$

The game $v^{G}$ is given by

$$
v^{G}(S)= \begin{cases}2 & \text { if }\{1,2,3,4\} \subseteq S, \\ 1 & \text { if }\{1,2\} \subseteq S \text { or }\{2,3\} \subseteq S \text { or }\{3,4\} \subseteq S, \text { and }\{1,2,3,4\} \nsubseteq S \\ 0 & \text { otherwise }\end{cases}
$$

for all $S \subseteq N$.

A game $v \in \mathrm{TU}^{N}$ is called monotonic if

$$
v(S) \leq v(T),
$$

for all $S, T \in 2^{N}$ with $S \subseteq T$. Minimum coloring games are integer valued nonnegative monotonic games as is seen in the following proposition. 
Proposition 2.1 Let $G=(N, E)$ be a graph. Then, $v^{G}$ is integer valued, nonnegative and monotonic.

Proof Integer valuedness and nonnegativity of $v^{G}$ are straightforward consequences of the definition. As for monotonicity, let $S, T \in 2^{N}$ with $S \subseteq T$. Note that if a minimum coloring for $G[S]$ uses $\chi(G[S])$ colors, then at most $|T \backslash S|$ additional colors are necessary for a minimum coloring of $G[T]$, i.e., $\chi(G[T]) \leq \chi(G[S])+|T \backslash S|$. As a consequence,

$$
v^{G}(S)=|S|-\chi(G[S]) \leq|S|-\chi(G[T])+|T \backslash S|=|T|-\chi(G[T])=v^{G}(T) .
$$

The core $C(v)$ of a game $v \in \mathrm{TU}^{N}$ is defined as the set of all allocations $x \in \mathbb{R}^{N}$ such that $\sum_{i \in N} x_{i}=v(N)$ (efficiency) and $\sum_{i \in S} x_{i} \geq v(S)$ for all $S \subseteq N$ (stability). Hence, the core consists of all possible allocations of $v(N)$ for which no coalition has an incentive to leave the grand coalition. Consequently, if the core is empty, then it is not possible to find a stable allocation of $v(N)$.

Okamoto (2008) characterized the core of minimum coloring games induced by perfect conflict graphs, where a graph $G=(N, E)$ is called perfect if

$$
\omega(G[S])=\chi(G[S])
$$

for all $S \subseteq N$. If a graph is not perfect, then it is called imperfect. In the characterization of the core provided below the notation of a characteristic vector is used. For $S \in$ $2^{N} \backslash\{\emptyset\}$, the characteristic vector $e^{S} \in \mathbb{R}^{N}$ is defined as

$$
e_{i}^{S}= \begin{cases}1 & \text { if } i \in S \\ 0 & \text { otherwise }\end{cases}
$$

for all $i \in N$.

Theorem 2.2 [cf. Okamoto (2008)] Let $G=(N, E)$ be a perfect graph. Then,

$$
C\left(v^{G}\right)=\operatorname{Conv}\left(\left\{e^{N \backslash S} \mid S \in \Omega(G)\right\}\right) .
$$

Chudnovsky et al. (2006) characterized perfect graphs. This characterization uses the notion of cycle graphs, where a cycle graph $C_{n}$ is a graph $G=(N, E)$ for which there exists a bijection $f:\{1, \ldots, n\} \rightarrow N$ such that

$$
E=\{\{f(i), f(i+1)\} \mid i \in\{1, \ldots, n-1\}\} \cup\{f(1), f(n)\} .
$$

An odd cycle graph is a cycle graph $C_{n}$ where $n$ is odd.

Theorem 2.3 [cf. Chudnovsky et al. (2006)] A graph is perfect if and only if it does not contain an odd cycle graph of length at least five, or a complement of such graph, as an induced subgraph. 
Example 2.2 Reconsider the conflict graph $G=(N, E)$ in Fig. 1. Since $G$ does not contain an odd cycle graph of length at least five, or a complement of such graph as an induced subgraph, we know from Theorem 2.3 that $G$ is a perfect conflict graph. Since the set $\Omega(G)$ of maximum cliques is given by

$$
\Omega(G)=\{\{1,3,5\},\{1,4,5\},\{2,4,5\}\}
$$

it follows from Theorem 2.2 that

$$
C\left(v^{G}\right)=\operatorname{Conv}(\{(0,1,0,1,0),(0,1,1,0,0),(1,0,1,0,0)\}) .
$$

\section{Simple minimum coloring games}

In this section we consider simple minimum coloring games. First, we characterize the class of conflict graphs inducing minimum coloring games that are simple. After that, we consider in more detail these conflict graphs and analyze the core of the induced minimum coloring games.

Recall that a game $v \in \mathrm{TU}^{N}$ is called simple if

(i) $v(S) \in\{0,1\}$ for all $S \subset N$,

(ii) $v(N)=1$,

(iii) $v$ is monotonic.

Let $\mathrm{SI}^{N}$ denote the class of all simple games with player set $N$. The following theorem gives a necessary and sufficient condition, in terms of the chromatic number, for a conflict graph to induce a simple minimum coloring game.

Theorem 3.1 Let $G=(N, E)$ be a graph. Then, $v^{G} \in S I^{N}$ if and only if $\chi(G)=$ $n-1$.

Proof (" $\Rightarrow$ ") Let $v^{G} \in \mathrm{SI}^{N}$. Then, $v^{G}(N)=1$ and consequently $\chi(G)=n-$ $v^{G}(N)=n-1$.

(" $\Leftarrow ")$ Let $\chi(G)=n-1$. Then, $v^{G}(N)=n-\chi(G)=1$. According to Proposition 2.1 $v^{G}$ is integer valued, nonnegative and monotonic, so in particular $v^{G}(S) \in\{0,1\}$ for all $S \subset N$, which implies $v^{G} \in \mathrm{SI}^{N}$.

Proposition 3.3 provides an upper bound on the number of maximum cliques for conflict graphs inducing simple games. This proposition also states that conflict graphs inducing simple games are perfect. In the proof of this proposition we use the following lemma, which gives the clique number and the chromatic number for odd cycle graphs of length at least five and their complements. The proof of this lemma is straightforward and therefore omitted.

Lemma 3.2 Let $k \in \mathbb{N}$ with $k \geq 2$. Then, $\omega\left(C_{2 k+1}\right)=2, \chi\left(C_{2 k+1}\right)=3$, $\omega\left(\overline{C_{2 k+1}}\right)=$ $k$ and $\chi\left(\overline{C_{2 k+1}}\right)=k+1$.

Proposition 3.3 Let $G=(N, E)$ be a graph. If $v^{G} \in S I^{N}$, then 
(i) $G$ is perfect,

(ii) $|\Omega(G)| \leq 2$.

Proof Let $v^{G} \in \mathrm{SI}^{N}$. Then, according to Theorem 3.1, $\chi(G)=n-1$.

Part (i): Suppose that $G$ is not perfect. Then, according to Theorem 2.3, there exists an $S \subseteq N$ such that $G[S]=C_{2 k+1}$ or $G[S]=\bar{C}_{2 k+1}$ with $k \geq 2$. Then, using Lemma 3.2, we have

$$
v^{G}(S)=|S|-\chi(G[S])=2 k+1-3=2 k-2 \geq 2>v^{G}(N)
$$

in case $G[S]=C_{2 k+1}$, or

$$
v^{G}(S)=|S|-\chi(G[S])=2 k+1-(k+1)=k \geq 2>v^{G}(N),
$$

in case $G[S]=\bar{C}_{2 k+1}$, which both contradict monotonicity of $v^{G}$. Hence, $G$ is perfect.

Part (ii): Since $G$ is perfect (see part (i)) and $v^{G}$ is simple, we have $\omega(G)=\chi(G)=$ $n-1$. Suppose $|\Omega(G)|>2$ and let $k, l$ and $m$ be three distinct vertices such that $N \backslash\{k\}, N \backslash\{l\}$ and $N \backslash\{m\}$ are maximum cliques of $G$. Since $G[N \backslash\{k\}]=K_{n-1}$ and $G[N \backslash\{l\}]=K_{n-1}$, we have

$$
\{\{i, j\} \mid i, j \in N, i \neq j\} \backslash\{k, l\} \subseteq E .
$$

Moreover, since $G[N \backslash\{m\}]=K_{n-1}$ and $\{k, l\} \subseteq N \backslash\{m\}$ we have $\{k, l\} \in E$. This implies $G=K_{n}$ which contradicts $\chi(G)=n-1$. Hence, $|\Omega(G)| \leq 2$.

Note that the conditions in Proposition 3.3 are only sufficient conditions and not necessary conditions. Consider for example the conflict graph in Fig. 4 in Sect. 4.1. This conflict graph is perfect and has two maximum cliques. However, this conflict graph does not induce a simple game because the value of the grand coalition in the induced minimum coloring game is 2 .

Due to the fact that conflict graphs are assumed to be connected on $N$, we may conclude from Theorem 3.1 that a conflict graph inducing a simple game has at least three vertices. Moreover, from the previous proposition in combination with Theorem 3.1 we may conclude that a conflict graph $G=(N, E)$ inducing a simple game has at least one and at most two maximum cliques of size $n-1$. So, there are two classes of conflict graphs on $n$ vertices inducing a simple game. The first class consists of the conflict graphs with one maximum clique of size $n-1$. Note that this class consists of $n-3$ different conflict graphs (up to isomorphism) ${ }^{1}$, because the vertex that is not in the maximum clique is adjacent to at least one vertex (because $G$ is assumed to be connected on $N$ ) and at most $n-3$ vertices (because otherwise there are two maximum cliques). For an illustration with six vertices, see Fig. 2a, b, c. The second class consists of the conflict graphs with two maximum cliques of size $n-1$. Note that

\footnotetext{
1 Two graphs $G=(N, E)$ and $G^{\prime}=\left(N^{\prime}, E^{\prime}\right)$ are called isomorphic if there exists a bijection $f: N \rightarrow N^{\prime}$ such that $\{u, v\} \in E$ if and only if $\{f(u), f(v)\} \in E^{\prime}$.
} 


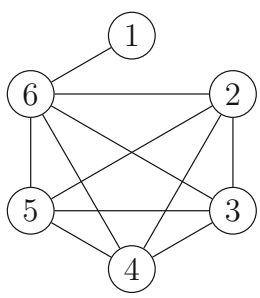

(a)

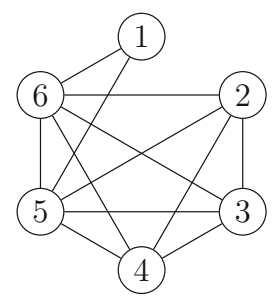

(b)

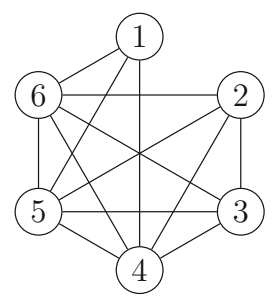

(c)

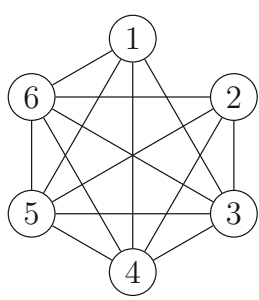

(d)

Fig. 2 All conflict graphs (up to isomorphism) on six vertices inducing a simple game

this class consists of a unique conflict graph (up to isomorphism), namely the conflict graph with exactly one pair of vertices not being adjacent. For an illustration with six vertices, see Fig. 2d. Hence, for given $n \geq 3$, there are $n-2$ different conflict graphs (up to isomorphism) on $n$ vertices inducing a simple game.

Using Theorem 2.2 and Proposition 3.3, one derives the following description of the core for simple minimum coloring games.

Corollary 3.4 Let $G=(N, E)$ be a graph and let $v^{G} \in S I^{N}$.

(i) If $\Omega(G)=\{N \backslash\{i\}\}$, then

$$
C\left(v^{G}\right)=e^{\{i\}}
$$

(ii) If $\Omega(G)=\{N \backslash\{i\}, N \backslash\{j\}\}$ with $i \neq j$, then

$$
C\left(v^{G}\right)=\operatorname{Conv}\left(\left\{e^{\{i\}}, e^{\{j\}}\right\}\right) .
$$

Example 3.1 Consider the conflict graph $G=(N, E)$ in Fig. 2a, b or c. Since $\Omega(G)=$ $\{\{2,3,4,5,6\}\}$ and $v^{G} \in \mathrm{SI}^{N}$, it follows from Corollary 3.4 that

$$
C\left(v^{G}\right)=\{(1,0,0,0,0,0)\}
$$

Next, consider the conflict graph $G=(N, E)$ in Fig. 2d. Since

$$
\Omega(G)=\{\{2,3,4,5,6\},\{1,3,4,5,6\}\},
$$

and $v^{G} \in \mathrm{SI}^{N}$, it follows from Corollary 3.4 that

$$
C\left(v^{G}\right)=\operatorname{Conv}(\{(1,0,0,0,0,0),(0,1,0,0,0,0)\}) .
$$

\section{Three-valued simple minimum coloring games}

In this section we characterize the class of conflict graphs inducing three-valued simple minimum coloring games. For this, a distinction is made between perfect and imperfect conflict graphs. After that, we characterize the core in terms of the underlying conflict graph for these games.

Musegaas et al. (2015) defined a game $v \in \mathrm{TU}^{N}$ to be three-valued simple if 
(i) $v(S) \in\{0,1,2\}$ for all $S \subset N$,

(ii) $v(N)=2$,

(iii) $v$ is monotonic.

Let $\operatorname{TSI}^{N}$ denote the class of all three-valued simple games with player set $N$. The following theorem gives a necessary and sufficient condition, in terms of the chromatic number, for a conflict graph to induce a three-valued simple game.

Theorem 4.1 Let $G=(N, E)$ be a graph. Then, $v^{G} \in \operatorname{TSI}^{N}$ if and only if $\chi(G)=$ $n-2 .^{2}$

Proof (" $\Rightarrow$ ") Let $v^{G} \in \mathrm{TSI}^{N}$. Then, $v^{G}(N)=2$ and consequently $\chi(G)=n-$ $v^{G}(N)=n-2$.

(" $\Leftarrow ")$ Let $\chi(G)=n-2$. Then, $v^{G}(N)=n-\chi(G)=2$. According to Proposition 2.1 $v^{G}$ is integer valued, nonnegative and monotonic, so in particular $v^{G}(S) \in\{0,1,2\}$ for all $S \subset N$, which implies $v^{G} \in \mathrm{TSI}^{N}$.

From now on, we distinguish between two classes of conflict graphs inducing three-valued simple minimum coloring games: perfect conflict graphs (Sect. 4.1) and imperfect conflict graphs (Sect. 4.2). For both classes, we consider in more detail the structure of these conflict graphs and the cores of the induced minimum coloring games.

\subsection{Three-valued simple minimum coloring games induced by perfect conflict graphs}

In this section we consider three-valued simple minimum coloring games induced by perfect conflict graphs. We show that for these games the core equals the vital core, introduced by Musegaas et al. (2015).

We start with providing an upper bound on the number of maximum cliques for perfect conflict graphs inducing three-valued simple games.

Proposition 4.2 Let $G=(N, E)$ be a perfect graph. If $v^{G} \in T S I^{N}$, then $|\Omega(G)| \leq 4$.

Proof Let $v^{G} \in \mathrm{TSI}^{N}$. Then, using Theorem 4.1, $\chi(G)=n-2$. Hence, due to the fact that $G$ is assumed to be connected on $N$, we have $n \geq 4$. Moreover, since $G$ is perfect, we have $\omega(G)=\chi(G)=n-2$, so at least two pairs of vertices are not adjacent in $G$. Without loss of generality we can assume that either $\{1,2\} \notin E$ and $\{3,4\} \notin E$, or $\{1,2\} \notin E$ and $\{2,3\} \notin E$. Therefore, we distinguish between the following two cases:

(a) Assume $\{1,2\} \notin E$ and $\{3,4\} \notin E$. Then the sets of vertices that can possibly form a maximum clique are

$$
\{T \subseteq N|| T \mid=n-2,\{1,2\} \nsubseteq T,\{3,4\} \nsubseteq T\}
$$

\footnotetext{
2 Note that Theorem 3.1 and Theorem 4.1 can be generalized for a more general class of integer valued, nonnegative and monotonic games.
} 
Therefore, a maximum clique is of the form

$$
N \backslash\{i, j\},
$$

with $i \in\{1,2\}$ and $j \in\{3,4\}$. Hence, there are only four sets of vertices that can possibly form a maximum clique, i.e., $|\Omega(G)| \leq 4$.

(b) Assume $\{1,2\} \notin E$ and $\{2,3\} \notin E$. Since $N \backslash\{2\}$ cannot form a clique, we know that there exists a pair of vertices $\{i, j\} \subseteq N \backslash\{2\}$ with $i \neq j$ and $\{i, j\} \notin E$. Hence, without loss of generality we can assume that $\{1,3\} \notin E,\{3,4\} \notin E$ or $\{4,5\} \notin E$ (the last case is only possible if $n \geq 5$ ).

If $\{3,4\} \notin E$, then we are back to case (a) and thus $|\Omega(G)| \leq 4$.

On the other hand, if $\{4,5\} \notin E$, then the sets of vertices that can possibly form a maximum clique are

$$
\{T \subseteq N|| T \mid=n-2,\{1,2\} \nsubseteq T,\{2,3\} \nsubseteq T,\{4,5\} \nsubseteq T\}
$$

Therefore, a maximum clique is of the form

$$
N \backslash\{2, j\},
$$

with $j \in\{4,5\}$. Hence, there are only two sets of vertices that can possibly form a maximum clique, i.e., $|\Omega(G)| \leq 2$.

Finally, if $\{1,3\} \notin E$, then the sets of vertices that can possibly form a maximum clique are

$$
\{T \subseteq N|| T \mid=n-2,\{1,2\} \nsubseteq T,\{1,3\} \nsubseteq T,\{2,3\} \nsubseteq T\}
$$

Therefore, a maximum clique is of the form

$$
N \backslash\{i, j\},
$$

with $i \neq j$ and $\{i, j\} \subseteq\{1,2,3\}$. Hence, there are only three sets of vertices that can possibly form a maximum clique, i.e., $|\Omega(G)| \leq 3$.

As one can see, in both cases $|\Omega(G)| \leq 4$.

Note that the condition in Proposition 4.2 is only a sufficient condition and not a necessary condition. Consider for example the conflict graphs in Fig. 2 in Sect. 3, which all are perfect and all have at most two maximum cliques. However, none of the conflict graphs induces a three-valued simple game because the value of the grand coalition in every induced minimum coloring game is 1 .

Due to the fact that conflict graphs are assumed to be connected on $N$, we may conclude from Theorem 4.1 that a conflict graph inducing a three-valued simple game has at least four vertices. Moreover, from the previous proposition we may also conclude that a perfect conflict graph $G=(N, E)$ inducing a three-valued simple game has at most four maximum cliques of size $n-2$. Figure 3 depicts all perfect conflict graphs (up to isomorphism) on four vertices inducing a three-valued simple game. All 


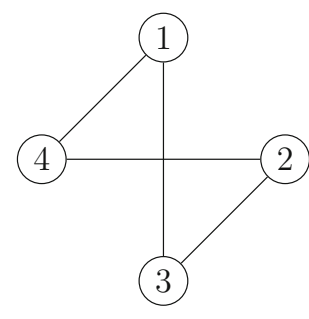

(a)

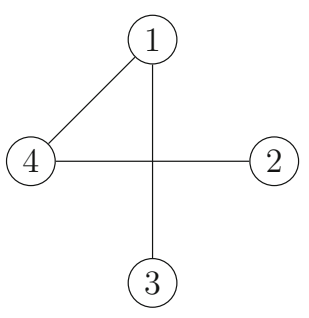

(b)

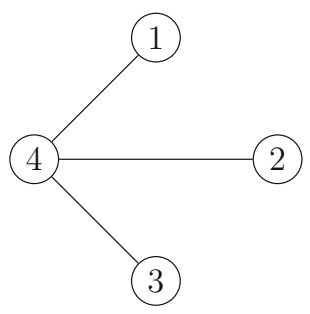

(c)

Fig. 3 All perfect conflict graphs (up to isomorphism) on four vertices inducing a three-valued simple game

conflict graphs have a clique number of two. The conflict graph in Fig. 3a has four maximum cliques and the conflict graphs in Fig. $3 \mathrm{~b}$, c have three maximum cliques.

Musegaas et al. (2015) analyzed the core of three-valued simple games. In particular, by introducing the concepts of vital players, primary vital players and secondary vital pairs, they defined the vital core for the class of permissible three-valued simple games. The vital core was shown to be a subset of the core for every permissible three-valued simple game. We summarize the relevant notions and results from Musegaas et al. (2015), and show that for the class of three-valued simple games induced by perfect conflict graphs, the core and the vital core coincide.

For $v \in \mathrm{TSI}^{N}$, the set of vital players is defined by

$$
\operatorname{Vit}(v)=\bigcap\{S \subseteq N \mid v(S)=2\} .
$$

Hence, the vital players are those players who belong to every coalition with value 2 . Using the concept of vital players, the following lemma provides a sufficient condition for emptiness of the core of a three-valued simple game.

Lemma 4.3 [cf. Musegaas et al. (2015)] Let $v \in \operatorname{TSI}^{N}$. If Vit $(v)=\emptyset$ or $v(N \backslash \operatorname{Vit}(v))>$ 0 , then $C(v)=\emptyset$.

From the previous lemma it follows that only the set of permissible three-valued simple games may have a non-empty core, where a game $v \in \mathrm{TSI}^{N}$ is called permissible if the following two conditions are satisfied:

(i) $\operatorname{Vit}(v) \neq \varnothing$,

(ii) $v(N \backslash \operatorname{Vit}(v))=0$.

For our further analysis of the core we can restrict attention to permissible three-valued simple games.

For a permissible game $v \in \operatorname{TSI}^{N}$, the reduced game $v_{r} \in \mathrm{TSI}^{\mathrm{Vit}(v)}$ is defined by

$$
v_{r}(S)=v(S \cup(N \backslash \operatorname{Vit}(v))),
$$

for all $S \subseteq \operatorname{Vit}(v)$. Note that a reduced game is also a three-valued simple game and allows for only one coalition with value 2, namely the grand coalition $\operatorname{Vit}(v)$. 
Table 1 Reduced game $v_{r}$ of the game $v$ in Example 4.1

\begin{tabular}{llllllll}
\hline$S$ & $\{1\}$ & $\{2\}$ & $\{3\}$ & $\{1,2\}$ & $\{1,3\}$ & $\{2,3\}$ & $\{1,2,3\}$ \\
\hline$v_{r}(S)$ & 0 & 0 & 0 & 1 & 1 & 0 & 2 \\
\hline
\end{tabular}

Interestingly, the core of a permissible three-valued simple game equals the core of the corresponding reduced game, when extended with zeros for all players outside the set of vital players. For a permissible game $v \in \mathrm{TSI}^{N}$, the set of primary vital players of $v$ is defined by

$$
\operatorname{PVit}(v)=\bigcap\left\{S \subseteq \operatorname{Vit}(v) \mid v_{r}(S) \in\{1,2\}\right\}
$$

and the set of secondary vital pairs of $v$ by

$\operatorname{SVit}(v)=\left\{\{i, j\} \subseteq \operatorname{Vit}(v) \backslash \operatorname{PVit}(v) \mid i \neq j,\{i, j\} \cap S \neq \emptyset\right.$ for all $S$ with $\left.v_{r}(S)=1\right\}$

Hence, the primary vital players are the vital players who belong to every coalition with value 1 or 2 in $v_{r}$. Similarly, the secondary vital pairs are the pairs of non-primary vital players such that for every coalition with value 1 in $v_{r}$, at least one player of such a pair belongs to the coalition. The vital core $V C(v)$ of a permissible game $v \in \mathrm{TSI}^{N}$ is defined by

$$
\begin{aligned}
V C(v)=\operatorname{Conv}\left(\left\{2 e^{\{i\}} \mid i \in \operatorname{PVit}(v)\right\}\right. \\
\\
\cup\left\{e^{\{i, j\}} \mid i \in \operatorname{PVit}(v), j \in \operatorname{Vit}(v) \backslash \operatorname{PVit}(v)\right\} \\
\left.\cup\left\{e^{\{i, j\}} \mid\{i, j\} \in \operatorname{SVit}(v)\right\}\right) .
\end{aligned}
$$

The following example illustrates the concepts introduced above.

Example 4.1 Let $N=\{1,2,3,4\}$ and consider the game $v \in \operatorname{TSI}^{N}$ given by

$$
v(S)= \begin{cases}2 & \text { if } S \in\{\{1,2,3\}, N\} \\ 1 & \text { if } S \in\{\{1,2\},\{1,3\},\{1,2,4\},\{1,3,4\}\} \\ 0 & \text { otherwise }\end{cases}
$$

Note that $v$ is permissible since $\operatorname{Vit}(v)=\{1,2,3\} \neq \emptyset$ and $v(N \backslash \operatorname{Vit}(v))=v(\{4\})=$ 0 . The corresponding reduced three-valued simple game $v_{r} \in \operatorname{TSI}^{\{1,2,3\}}$ is given in Table 1.

From the reduced game it follows that

$$
\operatorname{PVit}(v)=\{1\}
$$

and

$$
\operatorname{SVit}(v)=\{\{2,3\}\} .
$$


Therefore, the vital core of $v$ is given by

$$
V C(v)=\operatorname{Conv}\left(\left(\begin{array}{l}
2 \\
0 \\
0 \\
0
\end{array}\right),\left(\begin{array}{l}
1 \\
1 \\
0 \\
0
\end{array}\right),\left(\begin{array}{l}
1 \\
0 \\
1 \\
0
\end{array}\right),\left(\begin{array}{l}
0 \\
1 \\
1 \\
0
\end{array}\right)\right) .
$$

It can be shown that in this case we have $V C(v)=C(v)$.

For the game of Example 4.1 we have seen that the vital core equals the core. This need not be true for general three-valued simple games (see Example 3.4 in Musegaas et al. (2015)). In general, for a permissible game, the vital core is a subset of the core as is seen in the following theorem.

Theorem 4.4 [cf. Musegaas et al. (2015)] Let $v \in T S I^{N}$ be permissible. Then,

$$
V C(v) \subseteq C(v)
$$

The following theorem provides a characterization of the vital core for three-valued simple games induced by perfect conflict graphs.

Theorem 4.5 Let $G=(N, E)$ be a perfect graph. If $v^{G} \in T S I^{N}$, then

$$
V C\left(v^{G}\right)=\operatorname{Conv}\left(\left\{e^{N \backslash S} \mid S \in \Omega(G)\right\}\right) .
$$

Proof Let $v^{G} \in \mathrm{TSI}^{N}$. Then, using Theorem 4.1 and the fact that $G$ is perfect, we have $\omega(G)=\chi(G)=n-2$. In this proof we denote the intersection of all maximum cliques in $G$ by $\Omega_{G}$, i.e.,

$$
\Omega_{G}=\bigcap\{S \mid S \in \Omega(G)\}
$$

We divide the proof into proving the following four statements:

(i) $\operatorname{Vit}\left(v^{G}\right)=N \backslash \Omega_{G}$,

(ii) $v^{G}$ is permissible,

(iii) $\operatorname{PVit}\left(v^{G}\right)=\emptyset$,

(iv) $\operatorname{SVit}\left(v^{G}\right)=\{N \backslash S \mid S \in \Omega(G)\}$.

Note that if the statements (i) - (iv) all hold, then it immediately follows from the definition of the vital core that

$$
V C\left(v^{G}\right)=\operatorname{Conv}\left(\left\{e^{N \backslash S} \mid S \in \Omega(G)\right\}\right) .
$$

$\operatorname{Part}(i):\left[\operatorname{Vit}\left(v^{G}\right)=N \backslash \Omega_{G}\right]$

(" $\subset$ ") Let $i \in \Omega_{G}$, i.e., $i$ belongs to every maximum clique of $G$. This implies that if vertex $i$ is removed, then the clique number decreases with one. Therefore,

$$
v^{G}(N \backslash\{i\})=n-1-\chi(G[N \backslash\{i\}])=n-1-\omega(G[N \backslash\{i\}])
$$




$$
=n-1-(\omega(G)-1)=n-1-(\chi(G)-1)=n-1-(n-3)=2,
$$

where the second and the fourth equalities follow from the fact that $G$ is perfect. Hence, there exists an $S \subseteq N \backslash\{i\}$ such that $v^{G}(S)=2$, so $i \notin \operatorname{Vit}\left(v^{G}\right)$.

("つ") Let $i \in N \backslash \Omega_{G}$, i.e., there exists a maximum clique of $G$ to which $i$ does not belong. This implies that if vertex $i$ is removed, then the clique number does not change. Therefore,

$$
\begin{aligned}
v^{G}(N \backslash\{i\}) & =n-1-\chi(G[N \backslash\{i\}])=n-1-\omega(G[N \backslash\{i\}]) \\
& =n-1-\omega(G)=n-1-\chi(G)=n-1-(n-2)=1,
\end{aligned}
$$

where the second and the fourth equalities follow from the fact that $G$ is perfect. Moreover, from monotonicity of $v^{G}$ it follows that $v^{G}(S) \leq 1$ for all $S \subseteq N \backslash\{i\}$, so there does not exist an $S \subseteq N \backslash\{i\}$ with $v^{G}(S)=2$. As a consequence, for all $S \subseteq N$ with $v^{G}(S)=2$ we have $i \in S$ and thus $i \in \operatorname{Vit}\left(v^{G}\right)$.

Part (ii): $\left[v^{G}\right.$ is permissible]

Since $\omega(G)=n-2$, we know that $N$ cannot be a maxium clique and thus $\Omega_{G} \neq N$. Hence,

$$
\operatorname{Vit}\left(v^{G}\right)=N \backslash \Omega_{G} \neq \emptyset
$$

Moreover, since $\Omega_{G}$ is the intersection of all maximum cliques in $G$, we know that $\Omega_{G}$ forms a clique as well and thus $\chi\left(G\left[\Omega_{G}\right]\right)=\left|\Omega_{G}\right|$. As a consequence,

$$
v^{G}\left(N \backslash \operatorname{Vit}\left(v^{G}\right)\right)=v^{G}\left(\Omega_{G}\right)=0 .
$$

This implies that $v^{G}$ is permissible.

$\operatorname{Part}$ (iii): $\left[\operatorname{PVit}\left(v^{G}\right)=\emptyset\right]$

Since $\operatorname{Vit}\left(v^{G}\right)=N \backslash \Omega_{G}$, we know that for every vital player there exists a maximum clique of $G$ to which this player does not belong. As a consequence, for $i \in \operatorname{Vit}\left(v^{G}\right)$, we have

$$
\chi(G[N \backslash\{i\}])=\omega(G[N \backslash\{i\}])=\omega(G)=n-2,
$$

where the first equality follows from the fact that $G$ is perfect and the second equality follows from the fact that there exists a maximum clique of $G$ to which $i$ does not belong. Therefore,

$$
\begin{aligned}
v_{r}^{G}\left(\operatorname{Vit}\left(v^{G}\right) \backslash\{i\}\right) & =v^{G}\left(\operatorname{Vit}\left(v^{G}\right) \backslash\{i\} \cup\left(N \backslash \operatorname{Vit}\left(v^{G}\right)\right)\right)=v^{G}(N \backslash\{i\}) \\
& =n-1-\chi(G[N \backslash\{i\}])=n-1-(n-2)=1,
\end{aligned}
$$

for all $i \in \operatorname{Vit}\left(v^{G}\right)$. As a consequence, 


$$
\begin{aligned}
\operatorname{PVit}\left(v^{G}\right) & =\bigcap\left\{S \subseteq \operatorname{Vit}(v) \mid v_{r}^{G}(S) \in\{1,2\}\right\} \\
& \subseteq \bigcap\left\{\operatorname{Vit}\left(v^{G}\right) \backslash\{i\} \mid i \in \operatorname{Vit}\left(v^{G}\right)\right\}=\emptyset .
\end{aligned}
$$

$\operatorname{Part}(i v):\left[\operatorname{SVit}\left(v^{G}\right)=\{N \backslash S \mid S \in \Omega(G)\}\right]$

(" $\supset$ ") Let $S \in \Omega(G)$. Since $\omega(G)=n-2$, we can denote $N \backslash S=\{i, j\}$ with $i \neq j$. Note $\{i, j\} \cap \Omega_{G}=\emptyset$ and thus $\{i, j\} \subseteq \operatorname{Vit}\left(v^{G}\right)$. Moreover, since $\operatorname{PVit}\left(v^{G}\right)=\emptyset$, we have $\{i, j\} \subseteq \operatorname{Vit}\left(v^{G}\right) \backslash \operatorname{PVit}\left(v^{G}\right)$. Suppose $\{i, j\} \notin \operatorname{SVit}\left(v^{G}\right)$, then it follows from the definition of secondary vital pairs that there exists a $T \subseteq \operatorname{Vit}\left(v^{G}\right) \backslash\{i, j\}$ with $v_{r}^{G}(T)=1$. Since $T \subseteq \operatorname{Vit}\left(v^{G}\right) \backslash\{i, j\}$, we have

$$
\left(T \cup \Omega_{G}\right) \subseteq\left(\left(\operatorname{Vit}\left(v^{G}\right) \backslash\{i, j\}\right) \cup \Omega_{G}\right)=N \backslash\{i, j\}=S .
$$

Moreover, since $S$ forms a maximum clique in $G$, we have $\chi\left(G\left[T \cup \Omega_{G}\right]\right)=\left|T \cup \Omega_{G}\right|$ and thus

$$
\begin{aligned}
v_{r}^{G}(T) & =v^{G}\left(T \cup\left(N \backslash \operatorname{Vit}\left(v^{G}\right)\right)\right)=v^{G}\left(T \cup \Omega_{G}\right)=\left|T \cup \Omega_{G}\right|-\chi\left(G\left[T \cup \Omega_{G}\right]\right) \\
& =\left|T \cup \Omega_{G}\right|-\left|T \cup \Omega_{G}\right|=0,
\end{aligned}
$$

which contradicts $v_{r}^{G}(T)=1$. Hence, $\{i, j\} \in \operatorname{SVit}\left(v^{G}\right)$.

(" $\subset$ ") Let $\{i, j\} \in \operatorname{SVit}\left(v^{G}\right)$ and suppose $N \backslash\{i, j\}$ does not form a maximum clique in $G$, i.e., $\omega(G[N \backslash\{i, j\}])<n-2$. Then,

$$
\begin{aligned}
v_{r}^{G}\left(\operatorname{Vit}\left(v^{G}\right) \backslash\{i, j\}\right) & =v^{G}\left(\left(\operatorname{Vit}\left(v^{G}\right) \backslash\{i, j\}\right) \cup\left(N \backslash \operatorname{Vit}\left(v^{G}\right)\right)\right)=v^{G}(N \backslash\{i, j\}) \\
& =(n-2)-\chi(G[N \backslash\{i, j\}])=(n-2)-\omega(G[N \backslash\{i, j\}]) \\
& >(n-2)-(n-2)=0,
\end{aligned}
$$

where the penultimate equality again follows from the fact that $G$ is perfect. Consequently, using the fact that a reduced game allows for only one coalition with value 2 , namely the grand coalition $\operatorname{Vit}\left(v^{G}\right)$, we have $v_{r}^{G}\left(\operatorname{Vit}\left(v^{G}\right) \backslash\{i, j\}\right)=1$ which contradicts the assumption that $\{i, j\} \in \operatorname{SVit}\left(v^{G}\right)$.

Example 4.2 Consider the perfect conflict graph $G=(N, E)$ with $N=\{1, \ldots, 6\}$ as depicted in Fig. 4. Since $\Omega(G)=\{\{1,3,5,6\},\{1,4,5,6\}\}$ and $v^{G} \in \mathrm{TSI}^{N}$, it follows from Theorem 4.5 that

$$
V C\left(v^{G}\right)=\operatorname{Conv}(\{(0,1,0,1,0,0),(0,1,1,0,0,0)\}) .
$$

By combining Theorem 2.2 and Theorem 4.5 the general relation between the vital core and the core can be strengthened for three-valued simple minimum coloring games, i.e., for three-valued simple minimum coloring games induced by perfect conflict graphs the core equals the vital core.

Corollary 4.6 Let $G=(N, E)$ be a perfect graph. If $v^{G} \in T S I^{N}$, then

$$
C\left(v^{G}\right)=V C\left(v^{G}\right) .
$$




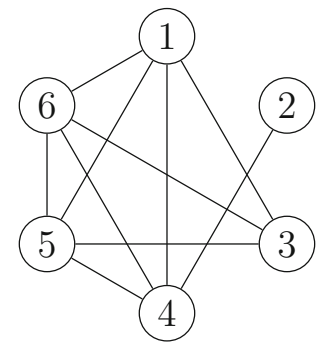

Fig. 4 The conflict graph of example 4.2

\subsection{Three-valued simple minimum coloring games induced by imperfect conflict graphs}

In this section we consider three-valued simple minimum coloring games induced by imperfect conflict graphs. First, we characterize this class of conflict graphs, after that we show that the induced minimum coloring games always have an empty core.

Note that all imperfect graphs have at least five vertices, because it must contain an odd cycle graph of length at least five, or a complement of such graph as an induced subgraph (cf. Theorem 2.3). The following theorem provides a necessary and sufficient condition for an imperfect conflict graph to induce a three-valued simple game. For this theorem, we use the notion of a dominating vertex. A vertex $i \in N$ is called dominating in $G$ if $\{j \mid j \in N,\{i, j\} \in E\}=N \backslash\{i\}$, i.e., if $i$ is adjacent to every other vertex.

Theorem 4.7 Let $G=(N, E)$ be an imperfect graph. Then, $v^{G} \in T S I^{N}$ if and only if there exists an $S \subseteq N$ such that $G[S]=C_{5}$ and all vertices outside $S$ are dominating.

Proof (" $\Leftarrow$ ") Let $S \subseteq N$ be such that $G[S]=C_{5}$ and let all vertices outside $S$ be dominating. Since all vertices in $N \backslash S$ are dominating, we have $G[N \backslash S]=K_{|N \backslash S|}$ and thus $\chi(G[N \backslash S])=n-5$. Moreover, since each vertex in $S$ is adjacent to each vertex in $N \backslash S$, we have

$$
\chi(G)=\chi(G[N \backslash S])+\chi(G[S])=n-5+3=n-2,
$$

where the second equality follows from Lemma 3.2. Consequently, using Theorem 4.1, we have $v^{G} \in \mathrm{TSI}^{N}$.

(" $\Rightarrow$ ") Let $v^{G} \in \mathrm{TSI}^{N}$. Since $G$ is not perfect, we know from Theorem 2.3 that there exists an $S \subseteq N$ such that $G[S]=C_{2 k+1}$ or $G[S]=\bar{C}_{2 k+1}$ with $k \geq 2$. Suppose $k>2$. Then, using Lemma 3.2, we have 


$$
v^{G}(S)=|S|-\chi(G[S])=2 k+1-3=2 k-2>2,
$$

in case $G[S]=C_{2 k+1}$, or

$$
v^{G}(S)=|S|-\chi(G[S])=2 k+1-(k+1)=k>2,
$$

in case $G[S]=\bar{C}_{2 k+1}$, which both contradict $v^{G}$ being a three-valued simple game. Hence, $k=2$ and thus $G[S]=C_{5}$ or $G[S]=\bar{C}_{5}$. Since $C_{5}$ and $\bar{C}_{5}$ are isomorphic to each other ${ }^{3}$. and thus both graphs have the same clique and chromatic number, we can conclude that $G[S]=C_{5}$.

Now, suppose $\chi(G[N \backslash S])<|N \backslash S|=n-5$. Then

$$
v^{G}(N)=n-\chi(G) \geq n-(\chi(G[S])+\chi(G[N \backslash S]))>n-(3+n-5)=2,
$$

which contradicts $v^{G}$ being a three-valued simple game. Hence, we may assume $\chi(G[N \backslash S])=n-5$ and thus $G[N \backslash S]=K_{|N \backslash S|}$. As a consequence, all players in $N \backslash S$ are mutually adjacent, i.e.,

$$
\{j \mid j \in N \backslash S,\{i, j\} \in E\}=(N \backslash S) \backslash\{i\},
$$

for all $i \in N \backslash S$.

Next, suppose $\{i, j\} \notin E$ for some $i \in S$ and $j \in N \backslash S$. Then, since there exists a vertex in $S$ that is not adjacent to a vertex in $N \backslash S$, those two players can receive the same color in the minimum coloring of $G$. Hence, $\chi(G)<\chi(G[S])+\chi(G[N \backslash S])$ and thus

$$
v^{G}(N)=n-\chi(G)>n-(\chi(G[S])+\chi(G[N \backslash S]))=n-(3+n-5)=2,
$$

which again contradicts $v^{G}$ being a three-valued simple game. Hence, we may assume that every player in $N \backslash S$ is adjacent to all players in $S$, i.e.,

$$
\{j \mid j \in S,\{i, j\} \in E\}=S,
$$

3 To see that $C_{5}$ and $\bar{C}_{5}$ are isomorphic to each other, consider the graphs $G=(N, E)$ and $G^{\prime}=\left(N^{\prime}, E^{\prime}\right)$ with $N=N^{\prime}=\{1, \ldots, 5\}$,

$$
E=\{\{1,2\},\{1,5\},\{2,3\},\{3,4\},\{4,5\}\},
$$

and

$$
E^{\prime}=\{\{1,3\},\{1,4\},\{2,4\},\{2,5\},\{3,5\}
$$

If one takes the bijection $f: N \rightarrow N^{\prime}$ with $f(1)=1, f(2)=3, f(3)=5, f(4)=2$ and $f(5)=4$, then $\{u, v\} \in E$ if and only if $\{f(u), f(v)\} \in E^{\prime}$ 


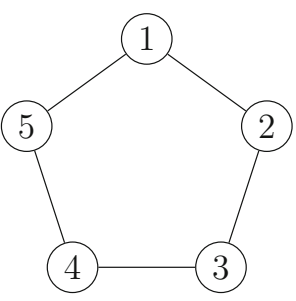

(a)

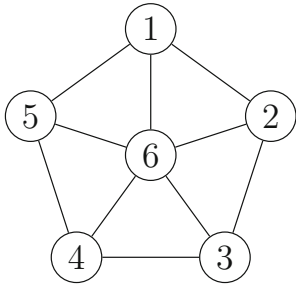

(b)

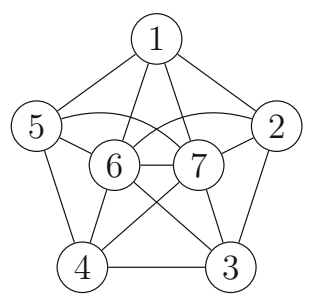

(c)

Fig. 5 All imperfect conflict graphs (up to isomorphism) on five, six or seven vertices inducing a threevalued simple game

for all $i \in N \backslash S$. Consequently,

$$
\{j \mid j \in N,\{i, j\} \in E\}=N \backslash\{i\},
$$

for all $i \in N \backslash S$, i.e., all vertices outside $S$ are dominating.

The previous theorem implies that, for given $n \geq 5$, the class of imperfect conflict graphs on $n$ vertices inducing a three-valued simple game consists of a unique conflict graph (up to isomorphism), namely the conflict graph that contains $C_{5}$ as an induced subgraph and all other vertices being dominated. Fig. 5 depicts all imperfect conflict graphs (up to isomorphism) on five, six and seven vertices inducing a three-valued simple game. For all these conflict graphs, the subgraph induced by $\{1,2, \ldots, 5\}$ is the cycle graph $C_{5}$ and all other vertices are dominating.

Using Theorem 4.7, the clique number and the number of maxiumum cliques for imperfect conflict graphs inducing three-valued simple games immediately follows, as is stated in the following corollary.

Corollary 4.8 Let $G=(N, E)$ be an imperfect graph. If $v^{G} \in T S I^{N}$, then $\omega(G)=$ $n-3$ and $|\Omega(G)|=5$.

For three-valued simple minimum coloring games induced by imperfect conflict graphs, the core is empty as is seen in the following theorem.

Theorem 4.9 Let $G=(N, E)$ be an imperfect graph. If $v^{G} \in T S I^{N}$, then $v^{G}$ is not permissible and thus $C\left(v^{G}\right)=\emptyset$.

Proof Let $v^{G} \in \mathrm{TSI}^{N}$. Then, Theorem 4.7 implies that there exists an $S \subseteq N$ such that $G[S]=C_{5}$. For $i \in S$, we have

$$
v^{G}(S \backslash\{i\})=4-\chi(G[S \backslash\{i\}])=4-2=2 .
$$

As a consequence,

$$
\operatorname{Vit}\left(v^{G}\right)=\bigcap\left\{S \subseteq N \mid v^{G}(S)=2\right\} \subseteq \bigcap\{S \backslash\{i\} \mid i \in S\}=\emptyset
$$

so $v^{G}$ is not permissible. Since only three-valued simple games that are permissible can have a non-empty core (see Lemma 4.3), we have $C\left(v^{G}\right)=\emptyset$. 
Open Access This article is distributed under the terms of the Creative Commons Attribution 4.0 International License (http://creativecommons.org/licenses/by/4.0/), which permits unrestricted use, distribution, and reproduction in any medium, provided you give appropriate credit to the original author(s) and the source, provide a link to the Creative Commons license, and indicate if changes were made.

\section{References}

Bietenhader T, Okamoto Y (2006) Core stability of minimum coloring games. Math Oper Res 31:418-431 Borm P, Hamers H, Hendrickx R (2001) Operations research games: a survey. TOP 9:139-199

Chudnovsky M, Robertson N, Seymour P, Thomas R (2006) The strong perfect graph theorem. Ann Math 164:51-229

Deng X, Ibaraki T, Nagamochi H (1999) Algorithmic aspects of the core of combinatorial optimization games. Math Oper Res 24:751-766

Deng X, Ibaraki T, Nagamochi H, Zang W (2000) Totally balanced combinatorial optimization games. Math Progr 87:441-452

Hamers H, Miquel S, Norde H (2014) Monotonic stable solutions for minimum coloring games. Math Progr 145:509-529

Musegaas M, Borm P, Quant M (2015). Three-valued simple games. CentER Discussion paper 2015-026, Tilburg University, Tilburg

von Neumann J, Morgenstern O (1944) Theory of games and economic behavior. Princeton University Press, Princeton

Okamoto Y (2003) Submodularity of some classes of the combinatorial optimization games. Math Methods Oper Res 58:131-139

Okamoto Y (2008) Fair cost allocations under conflicts -a game-theoretic point of view-. Discrete Optim 5:1-18

Pardalos P, Mavridou T, Xue J (1999) The graph coloring problem: a bibliographic survey. In: Du D, Pardalos P (eds) Handbook of combinatorial optimization. Springer, USA, pp 1077-1141

Randerath B, Schiermeyer I (2004) Vertex colouring and forbidden subgraphs - a survey. Graphs and Combinatorics 20:1-40

Shapley L, Shubik M (1954) A method for evaluating the distribution of power in a committee system. Am Polit Sci Rev 48:787-792 